above $1.5 \mu \mathrm{V}^{2}$ for longer than 1 s. Each EEG channel was processed separately and the final outcome was considered to be an activity burst if a burst was detected in 2 or more channels.

The method was tested on a database of 24 babies born before 30 weeks gestation (avg $26.5+-1.7$ weeks). For each baby 10 minutes of 8-channel EEG signal was analysed.

Results Agreement with the expert burst annotations was on average $77.2 \%$ over the 24 subjects (Min:59.6\%, Max:90.3\%, stddev:8.5\%). Most errors consisted of disagreement over the precise start and end points of a burst.

Conclusions Automatic burst detection has been applied for the first time to a large database of preterm 8-channel EEG. Promising results were obtained for automated EEG interpretation. Future work will attempt to reduce the error by use of more sophisticated methods to merge the per-channel detections.

\section{RELATIONSHIP BETWEEN ACUTE KIDNEY INJURY (AKI) USING AKI NETWORK CRITERIA AND BRAIN MRI FINDINGS IN ASPHYXIATED NEWBORNS AFTER THERAPEUTIC HYPOTHERMIA}

doi:10.1136/archdischild-2012-302724.0334

'S Sarkar, 'B Jordan, 2 B Bhagat, 3.JR Bapuraj, 'R Dechert, "4D Selewski. 'Department of Pediatrics, Division of Neonatal-Perinatal Medicine, University of Michigan Health System; ${ }^{2}$ Pediatrics, St Joseph Mercy Hospital; ${ }^{3}$ Department of Radiology; ${ }^{4}$ Department of Pediatrics, Division of Pediatric Nephrology, University of Michigan Health System, Ann Arbor, MI, USA

We hypothesized that hypoxic-ischemic lesion on brain MRI would differ between infants with AKI compared to those without AKI following cooling.

Methods 88 consecutively cooled infants who had brain MRI were reviewed. All infants had renal function assessed before the start of cooling (baseline); at 24, 48, and 72h through cooling; and then on day 5 or 7 of life. Injuries to both basal nuclei and cortex on MRI were considered severely abnormal.

Results AKI was found in 34 (39\%) of 88 infants with 15, 7, and 12 fulfilling AKI network criteria for stage I, II, and III, respectively. Hypoxic-ischemic lesion on brain MRI was present in 50 infants. In 26 infants (AKI 14, no AKI 12), MRI was severely abnormal.

Abnormal MRI was more frequent in the AKI group (AKI 25 of $34,73 \%$ versus No AKI 25 of $54,46 \%, p=0.012$, OR $3.2,95 \%$ CI 1.3-8.2). Multivariate analysis identified only the AKI ( $\mathrm{p}=0.032$, OR $2.9,95 \%$ CI 1.1-7.6), and chest compression for resuscitation to be independently associated with primary outcome.

Severely abnormal MRI were similar between infants with stage III and stage II AKI (stage III 3 of $12,25 \%$ versus stage II 3 of $7,43 \%$; $\mathrm{p}=0.617$ ), or stage I AKI (stage III 3 of $12,25 \%$ versus stage I 8 of 15 , $53 \%$; $=0.238)$.

Conclusions AKI is independently associated with the presence of hypoxic-ischemic lesions on post-cooling brain MRI. However, the severity of AKI did not correlate with the severity of brain MRI abnormalities

\section{BRAIN PLASTICITY AFTER PRETERM BIRTH: AN EEG STUDY OF AUDITORY PROCESSING}

doi:10.1136/archdischild-2012-302724.0335

'A Darque, 'R HaVinh Leuchter, ${ }^{2} \mathrm{TA}$ Rihs, ${ }^{3} \mathrm{~F}$ Lazeyras, ${ }^{3} \mathrm{C}$ Caballero, ${ }^{2} \mathrm{CM}$ Michel, ${ }^{1} \mathrm{PS}$ Hüppi. 'Department of Pediatrics, University Hospitals of Geneva; ${ }^{2}$ Functional Brain Mapping Laboratory, Dept. of Fundamental Neurosciences, University of Geneva; ${ }^{3}$ Department of Radiology, University Hospitals of Geneva, Geneva, Switzerland

Background and Aims Premature birth has an impact on brain maturation that can be measured at term equivalent age (TEA) with neuroimaging techniques. The aim of our study is to determine the neural pathways and processes that are activated in term babies and preterm infants $(\mathrm{GA}<32 \mathrm{wks})$ at term after listening to their mother's voice and a stranger's voice with EEG and fMRI techniques. Our secondary aim is to differentiate innate (genetically determined) and acquired (determined by experience) networks. Here we present the results of the EEG analysis of the preterm recordings.

Methods High-density (109-channel) recordings were performed for subsequent event-related potentials (ERPs) analysis on newborns while listening to their mother's voice and the voice of an unknown woman saying a short phrase. Two groups were tested: premature newborns tested at TEA (GA:28.7wks) and full term controls (GA:40wks).

Results For preterm babies, the ERP results showed significant differences on left temporal electrodes when they listened to their mother's voice compared to a stranger's voice with an increased negativity at $100 \mathrm{~ms}$ post voice onset ( $\mathrm{t}$-test; $\mathrm{p}<0.05)$. At later stages of voice processing, significant differences were found between 220-320ms with increased positivity for the mothers voice over right temporal electrodes.

Conclusions By showing specific activation in preterm babies at term when they listen to their mother's voice, our results suggest that the maturation of the auditory network can be influenced by these early experiences resulting in an early differentiation between their mother's voice and the voice of a stranger.

\section{ARTERIAL SPIN LABELING MAGNETIC RESONANCE IMAGING TO EVALUATE PERINATAL ARTERIAL ISCHEMIC STROKE}

doi:10.1136/archdischild-2012-302724.0336

'JB De Vis, ${ }^{2} \mathrm{KJ}$ Kersbergen, ${ }^{1} \mathrm{ET}$ Petersen, ${ }^{1} \mathrm{~J}$ Hendrikse, ${ }^{2} \mathrm{~T}$ Alderliesten, ${ }^{2} \mathrm{~F}$ Groenendaal ${ }^{2} \mathrm{LS}$ de Vries, ${ }^{2} \mathrm{MJNL}$ Benders. 'Radiology, University Medical Center Utrecht; ${ }^{2}$ Neonatology, Wilhelmina Childrens' Hospital/UMCU, Utrecht, The Netherlands

Background and Aim Studies performed in infants with perinatal arterial ischemic stroke (PAIS) have shown relations between initial neuro-imaging and neurodevelopmental outcome. However, not all variation in outcome can be explained. It is known from adult stroke studies that (luxury) perfusion of the stroke area is related to outcome. In this study, Arterial Spin Labeling (ASL) MR imaging was used to evaluate (luxury) perfusion in infants with PAIS.

Methods Conventional and ASL MR images (3T) were acquired of three PAIS patients 3-5 days after the ischemic event. Near Infrared Spectroscopy was used to monitor cerebral oxygenation. Follow-up MR imaging was conducted 2-16 weeks after the event.

Results A lower perfusion signal was measured in all infants in the area corresponding with the diffusion-restricted area on the diffusion-weighted images. Furthermore, in one infant, luxury perfusion was visualised in the cortex of the affected area. Measurements of volume flow (Phase-Contrast MR Angiography) and cerebral oxygenation were in agreement with this, suggesting an ischemiainduced vasodilatation. Follow-up ASL MR images in this infant showed a partly recovered perfusion. Initial [fig. 1A] and follow-up ASL images [Fig. 1B] of this infant are shown.

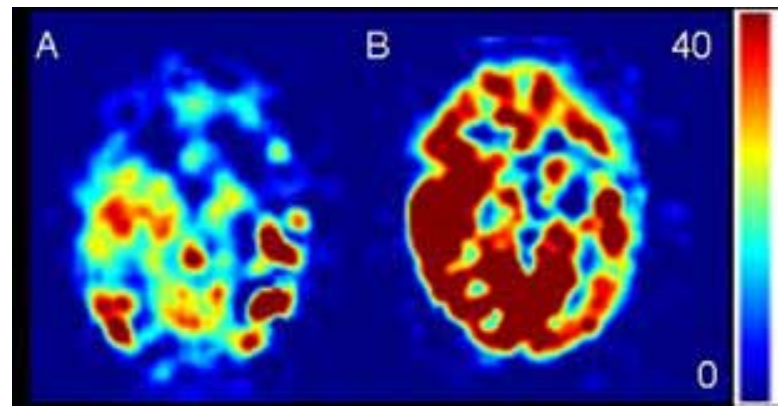

Abstract 336 Figure 1 